\title{
Complement 3 glomerulonephritis in rheumatoid arthritis: A case report and follow-up
}

\author{
XIN WANG, LU HAN, HUIFANG LI and DONGMEI ZHANG \\ Department of Nephrology, The Second Hospital of Jilin University, Changchun, Jilin 130041, P.R. China
}

Received December 10, 2017; Accepted June 8, 2018

DOI: $10.3892 /$ etm.2018.6476

\begin{abstract}
Glomerulonephritis (GN) caused by rheumatoid arthritis may manifest as various pathological types; however, to the best of our knowledge, rheumatoid arthritis-associated complement 3 (C3)-GN has not been reported by any previous studies. C3GN is caused by dysregulation of the alternative pathway of complements, which is completely different from activation of the classic pathway of a typical autoimmune disease to cause renal damage. The present study describes a patient with a history of rheumatoid arthritis for 18 years, who presented with edema, proteinuria, hematuria, hypoproteinemia and a hypocomplementemic state. The pathological diagnosis was $\mathrm{C} 3 \mathrm{GN}$ based on histological examination of a renal biopsy specimen. Hormone treatment on its own appeared to be effective and achieved complete clinical remission, while the follow-up of the condition remained stable.
\end{abstract}

\section{Introduction}

Rheumatoid arthritis (RA) frequently involves other organs, including the lung, kidney, eyes and skin, apart from the joints. RA-induced kidney damage may have various clinical and pathological manifestations, but to date, RA-induced complement 3 glomerulonephritis (C3GN) has not been reported, to the best of our knowledge. C3GN is caused by dysregulation of the alternative pathway of complement diseases (1). C3GN may be caused by acquired or congenital defects. Acquired C3GN refers to the antibodies in the body against activated complement factors, including the $\mathrm{C} 3$ nephritis factor or anti-H factor antibodies, which continually activate the alternative pathway

Correspondence to: Dr Dongmei Zhang, Department of Nephrology, The Second Hospital of Jilin University, 218 Ziqiang Road, Changchun, Jilin 130041, P.R. China

E-mail: dmzhang@jlu.edu.cn

Abbreviations: C3, complement 3; GN, glomerulonephritis; RA, rheumatoid arthritis; Ig, immunoglobulin; AAV, ANCA-associated vasculitis

Key words: rheumatoid arthritis, C3 glomerulonephritis, follow-up, case report of complements. $\mathrm{C} 3 \mathrm{GN}$ caused by congenital defects refers to a genetic abnormality or gene mutation in the alternative pathway of complements that causes excessive activation (2). Studies have indicated that monoclonal immunoglobulinopathies may also activate the alternative pathway of complements and thus induce C3GN $(3,4)$. In addition, a study by Alexander et al (5) on C3GN and autoimmune disease suggests that an autoimmune milieu may serve as a trigger for the development of $\mathrm{C} 3 \mathrm{GN}$ in patients who carry $\mathrm{C} 3$ glomerulopathy-associated risk alleles by leading to dysregulation of the alternative pathway of complements. The study also suggested that the short-term prognosis of C3GN associated with autoimmune disorders is excellent. The present case study reports on renal involvement in a patient with RA after 18 years, who was diagnosed with $\mathrm{C} 3 \mathrm{GN}$ through renal biopsy. The patient's treatment, follow-up and outcome are presented.

\section{Case report}

A 63-year-old Chinese woman presented at the Second Hospital of Jilin University (Changchun, China) in January 2015 due to polyarthritis with pain, stiffness and swelling for 18 years, as well as acute bilateral leg swelling and urinary abnormalities for 1 week. She had been diagnosed as having RA 18 years ago when she presented with spontaneous symmetrical polyarthritis involving the small joints of her hands and knee joint with significant morning stiffness, and she received symptomatic treatment (the specific treatment is unknown). Subsequently, she achieved remission of the symptoms, but the symptoms of joint pain and swelling were recurrent, so she took medication, including analgesics, Chinese herbal supplements and various home remedies; however, her medical condition did not stabilize. The patient had stopped using these herbal supplements for $>1$ year prior to her presentation at hospital. One week prior to her presentation at hospital, she had bilateral pitting leg edema with no obvious predisposing causes. The urine analysis revealed hematuria $3+$ and proteinuria $3+$ and thus, the patient was scheduled for further treatment. The patient's medical history included cholecystitis for 6 years, which was stable, and bilateral inguinal hernia 3 years previously, which was surgically treated. The patient had no history of hypertension, diabetes or coronary disease, no known drug or food allergy, no known family history of hypertension, diabetes, autoimmune disease or kidney disease, and she was a non-smoker and did not consume any alcohol. The physical 


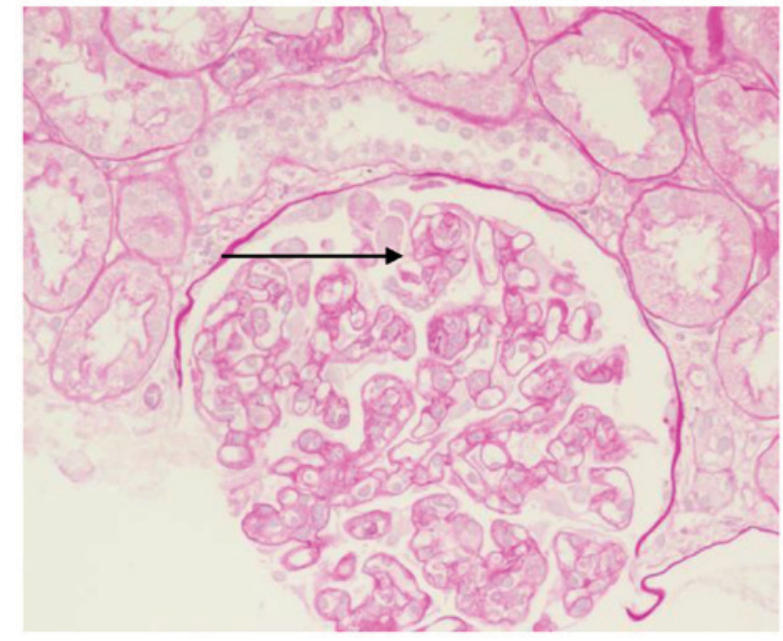

Figure 1. Light microscopy: The arrow indicates diffuse proliferation of glomerular endothelial cells and where the opening of the capillary loops was restricted (periodic acid-Schiff stain; magnification, x200).

examination revealed the following: Underarm temperature, $36.5^{\circ} \mathrm{C}$; pulse, 80 beats/min; respiratory rate, 18 breaths $/ \mathrm{min}$; blood pressure, 145/85 $\mathrm{mmHg}$; height, $153 \mathrm{~cm}$; and weight, $48 \mathrm{~kg}$. She had mucosal edema of the face with anemic palpebral conjunctiva, and mild dropsy of the bilaretal eyelids and legs. Her hands had an ulnar deviation and swan-neck deformity on the first to fourth fingers. The proximal interphalangeal joint, metacarpophalangeal joint and wrist joint had tenderness. Her wrist, elbow and knee joints had a slightly limited range of motion. No subcutaneous nodules were observed on the joints of her four extremities. The laboratory test results are presented in Table I. Apart from serum creatinine and urea nitrogen, which are normal values, the remainder of the results in Table I are abnormal. The chest computed tomography scan revealed bilateral pleural effusion, predominantly on the left side. The bone marrow morphology test results indicated hyperplastic anemia with an iron deficiency (cellular iron and exocellular iron were negative). Histological analysis of the renal biopsy indicated the following: 15 whole glomeruli were observed, part of the glomerular volume was increased, 3 glomeruli were globally sclerotic, 2 cells were fibrotic, 1 small cellular crescent formation was present and 2 capillary loops exhibited segmental fibroid necrosis. Glomerular endothelial cells exhibited diffuse hyperplasia, certain capillary loops displayed limited opening (Fig. 1), renal tubular epithelial cells were diffuse with granular degeneration, tubular focal atrophy was present, protein and red blood cell cast was visible, mild edema was observed in the renal interstitium, small focal fibrosis and focal inflammatory-cell infiltration were present, an inflammatory cell cluster was observed and the prevailing cell type was mononuclear cells (Fig. 2). The small arteries were unremarkable. Periodic-acid methenamine silver and Masson staining indicated no spikes or double tracking, and there was a deposition of a segmental eosinophilic substance in the subendothelial area (Fig. 3). Congo red staining was negative (data not shown). Immunofluorescence microscopy revealed bright $\mathrm{C} 3$ staining $(2+)$ in the mesangium and granular deposition along the capillary walls (Fig. 4), while

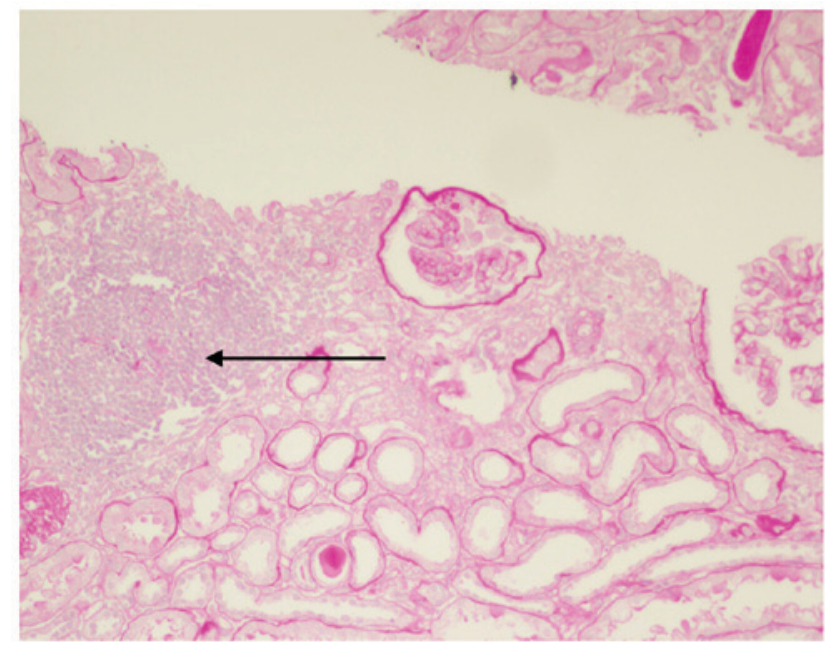

Figure 2. Light microscopy. The arrow indicates the tubulointerstitial lesions and 1 inflammatory cell cluster (periodic acid-Schiff stain; magnification, x100).

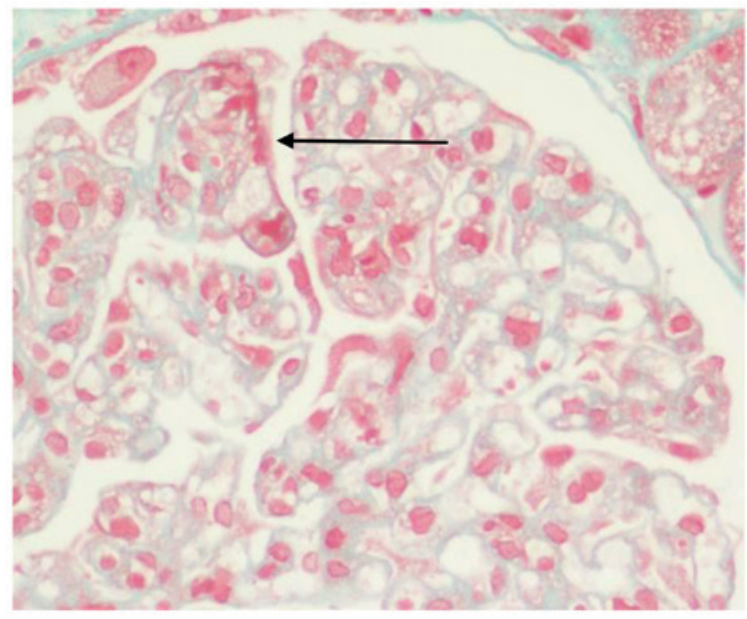

Figure 3. Light microscopy. The arrow indicates where the immune complexes were segmentally deposited on the subendothelial area (Masson trichrome stain; magnification, $\mathrm{x} 400$ ).

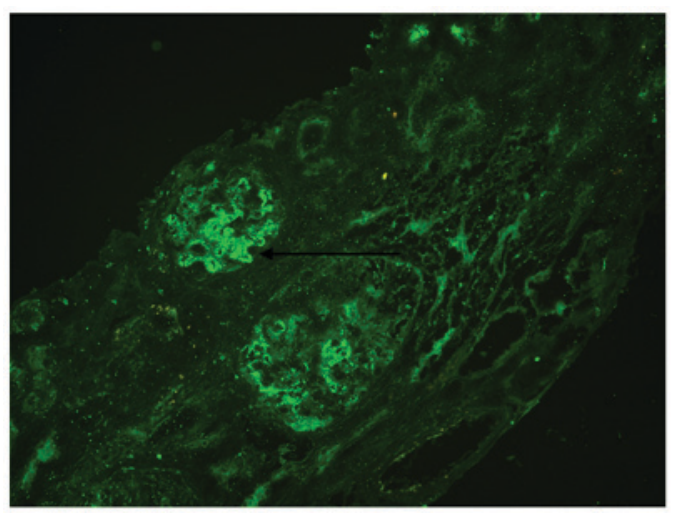

Figure 4. Immunofluorescence microscopy. The arrow indicates bright $\mathrm{C} 3$ staining in the mesangium and granular deposition along the capillary walls (magnification, $\mathrm{x} 400$ ).

immunofluorescent reactivity was negative for immunoglobulin (Ig)G, IgM, IgA, C4, C1q and fibrillarin (results not 
Table I. Patient's laboratory examination results.

\begin{tabular}{|c|c|c|}
\hline Parameters & Value & Reference range \\
\hline Proteinuria & $3+$ & Negative \\
\hline 24-h urine protein $(\mathrm{g})$ & 4.46 & $0-0.15$ \\
\hline Urine RBC count (/HPF) & 52.0 & $0.0-5.0$ \\
\hline $\mathrm{Hb}(\mathrm{g} / \mathrm{l})$ & 56 & $115-150$ \\
\hline $\mathrm{RBC}$ count $\left(\mathrm{x} 10^{12} / \mathrm{l}\right)$ & 2.63 & $3.80-5.10$ \\
\hline Mean corpuscular volume (fl) & 71.9 & $82.0-100.0$ \\
\hline Mean corpuscular $\mathrm{Hb}(\mathrm{pg})$ & 21.3 & $27.0-34.0$ \\
\hline Mean corpuscular Hb concentration (g/l) & 296.0 & $316.0-354.0$ \\
\hline $\mathrm{TP}(\mathrm{g} / \mathrm{l})$ & 64.7 & $65-85$ \\
\hline Albumin (g/l) & 31.1 & $40-55$ \\
\hline Total cholesterol (mmol/l) & 8.75 & $2.9-5.17$ \\
\hline LDL cholesterol (mmol/l) & 4.13 & $2.70-3.40$ \\
\hline Serum creatinine $(\mu \mathrm{mol} / 1)$ & 95 & 44-106 \\
\hline Urea nitrogen (mmol/l) & 7.08 & $1.79-7.14$ \\
\hline Anti-CCP level (RU/ml) & 142.0 & $0-5$ \\
\hline AKA & Positive & Negative \\
\hline APF & Weakly positive & Negative \\
\hline P-ANCA & Positive & Negative \\
\hline ANCA-MPO (RU/ml) & $<20$ & $0-20$ \\
\hline ANA ratio & 1:320; homogeneous & $<1: 100$ \\
\hline $\mathrm{RF}(\mathrm{IU} / \mathrm{ml})$ & $<20$ & $0.0-20.0$ \\
\hline ESR (mm) & 102.0 & $<20$ \\
\hline CRP (mg/l) & 2.37 & $0.00-0.80$ \\
\hline C3 (mg/dl) & 68.90 & $79-152$ \\
\hline C4 (mg/dl) & 12.60 & $16-38$ \\
\hline $\operatorname{IgA}(\mathrm{g} / \mathrm{l})$ & 5.91 & $0.82-4.53$ \\
\hline TSH (mIU/l) & $>150.000$ & $0.55-4.78$ \\
\hline FT3 (pmol/l) & 1.36 & $3.5-6.5$ \\
\hline FT4 (pmol/l) & 4.25 & $11.5-22.7$ \\
\hline TPOAb (U/ml) & 247.40 & $0-60$ \\
\hline $\operatorname{TgAb}(\mathrm{U} / \mathrm{ml})$ & 127 & $0-60$ \\
\hline TT3 (nmol/1) & 0.36 & $0.92-2.79$ \\
\hline TT4 (nmol/l) & 24.80 & $58.1-140.6$ \\
\hline Ferritin (ng/ml) & 5.10 & $11.0-306.8$ \\
\hline
\end{tabular}

RBC, red blood cell; Hb, hemoglobin; TP, total protein; LDL, low-density lipoprotein; CCP, cyclic citrullinated peptide; AKA, anti-keratin antibody; APF, anti-perinuclear factor autoantibody; P-ANCA, perinuclear anti-neutrophil cytoplasmic antibody; MPO, myeloperoxidase; ANA, anti-nuclear antibody; RF, rheumatoid factor; ESR, erythrocyte sedimentation rate; CRP, C-reactive protein; C3, complement 3; C4, complement 4; IgA, immunoglobulin A; FT3, free triiodothyronine; FT4, free thyroxine; TSH, thyroid-stimulating hormone; TPOAb, anti-thyroid peroxidase antibody; TgAb, anti-thyroglobulin antibody; TT3, total triiodothyronine acid; TT4, total thyroxine; HPF, high-power field.

shown). Electron microscopy indicated glomerular mesangial cell proliferation and mild hyperplasia of the mesangial matrix, diffuse endothelial cell proliferation, a widened inner tectorium of the basal membrane segment, electron-dense deposits in the subendothelial, mesangial and paramesangial area, fusion of most foot processes and increased lysosome in renal tubular epithelial cells; part of the microvilli exhibited shedding, atrophy, mild edema of the tubular interstitium, a small amount of lymphocytes and mononuclear-cell infiltration with collagen fiber hyperplasia (Figs. 5 and 6). The clinical diagnoses were RA, C3GN, hypothyroidism and iron-deficiency anemia. The patient was treated with a daily dose of prednisone acetate $(40 \mathrm{mg})$ combined with a lipid-lowering drug, anticoagulant and supplements of thyroxine and iron. The patient's follow-up results are presented in Table II. These included changes in each index during the follow-up (including urinary protein, $24 \mathrm{~h}$ urinary protein quantification, erythrocyte, hemoglobin, albumin and complement). It was observed that the $24 \mathrm{~h}$ urinary protein quantification rapidly decreased, the urine red blood cells gradually decreased, the serum albumin gradually increased and hemoglobin gradually increased to normal. The patient now receives small doses of prednisone acetate maintenance therapy (10 mg per day). 
Table II. Follow-up results.

\begin{tabular}{|c|c|c|c|c|c|c|}
\hline Parameters & 1 month & 2 months & 4 months & 8 months & 14 months & 18 months \\
\hline Proteinuria & $2+$ & $3+$ & $2+$ & $1+$ & $1+$ & $1+$ \\
\hline 24-h urine protein $(\mathrm{g})$ & & 3.53 & 0.5 & & 0.35 & 0.34 \\
\hline Urine RBC count (/HPF) & 27.5 & 13.3 & 4 & 4.5 & 2.7 & 2.8 \\
\hline $\mathrm{Hb}(\mathrm{g} / \mathrm{l})$ & 86 & 103 & 109 & 120 & 148 & 155 \\
\hline RBC count, $\left(\times 10^{12} / 1\right)$ & 3.51 & 3.87 & 4.34 & 4.57 & 4.85 & 5.08 \\
\hline $\mathrm{TP}(\mathrm{g} / \mathrm{l})$ & 55.6 & 52.1 & 63.1 & 55.6 & 70.8 & 67.2 \\
\hline Albumin (g/l) & 29 & 30 & 36.7 & 34.3 & 39.7 & 37.2 \\
\hline FT3 (pmol/l) & 1.89 & 2.32 & 3.31 & & 3.98 & 4.78 \\
\hline FT4 (pmol/l) & 12.21 & 15.78 & 22.47 & & 23.73 & 22.74 \\
\hline TSH (mIU/l) & 53.408 & 26.229 & 10.985 & & 6.534 & 5.576 \\
\hline Anti-CCP (RU/ml) & & & & & 88 & 18 \\
\hline $\mathrm{C} 3(\mathrm{mg} / \mathrm{dl})$ & & & & & & 76.00 \\
\hline $\mathrm{C} 4(\mathrm{mg} / \mathrm{dl})$ & & & & & & 14.80 \\
\hline $\mathrm{RF}(\mathrm{IU} / \mathrm{ml})$ & & & $<20$ & $<20$ & $<20$ & $<20$ \\
\hline AKA & & & & & Negative & Negative \\
\hline
\end{tabular}

RBC, red blood cell; Hb, hemoglobin; HPF, high-power field; TP, total protein; FT3, free triiodothyronine; FT4, free thyroxine; TSH, thyroid-stimulating hormone; $\mathrm{CCP}$, cyclic citrullinated peptide; $\mathrm{C} 3$, complement $3 ; \mathrm{C} 4$, complement 4 ; $\mathrm{RF}$, rheumatoid factor; AKA, anti-keratin antibody.

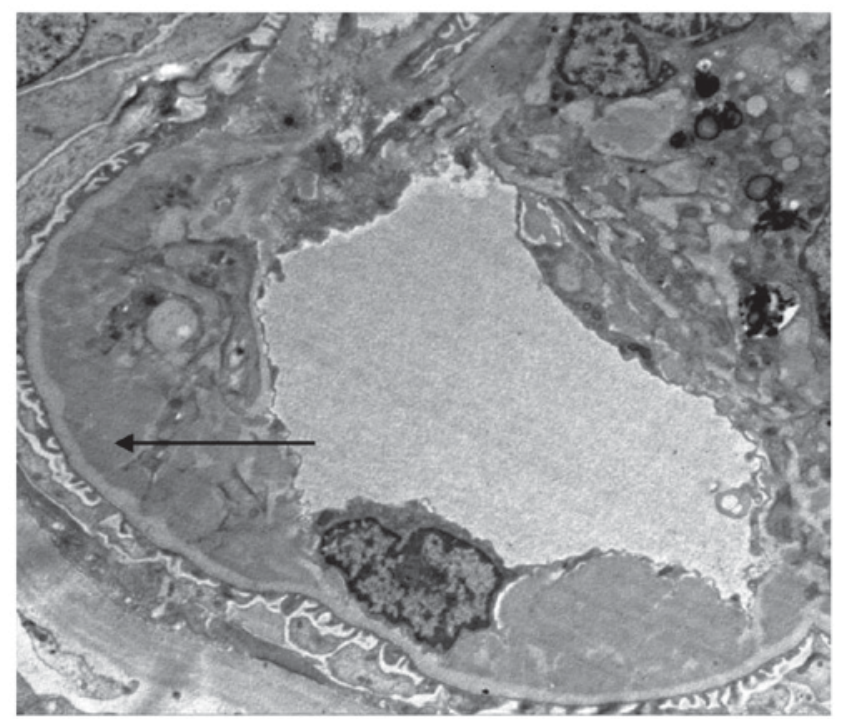

Figure 5. Electron microscopy. The arrow indicates the electron-dense deposits in the subendothelial area (magnification, x6,000).

\section{Discussion}

C3 glomerulopathy has been gradually recognized in recent years. Fakhouri et al (1) proposed the concept of C3 nephropathy for the first time in 2010. Subsequently, the definition of C3 nephropathy was further substantiated and revised by multidisciplinary experts in 2012 (2). To date, numerous studies on C3 glomerulopathy from China and abroad have been published $(6,7)$. C3 nephropathy results from dysregulation of the alternative pathway of $\mathrm{C} 3$, and the major pathological characteristics are the deposition of complement $\mathrm{C} 3$ on glomeruli, with or without minimal Ig deposition and without

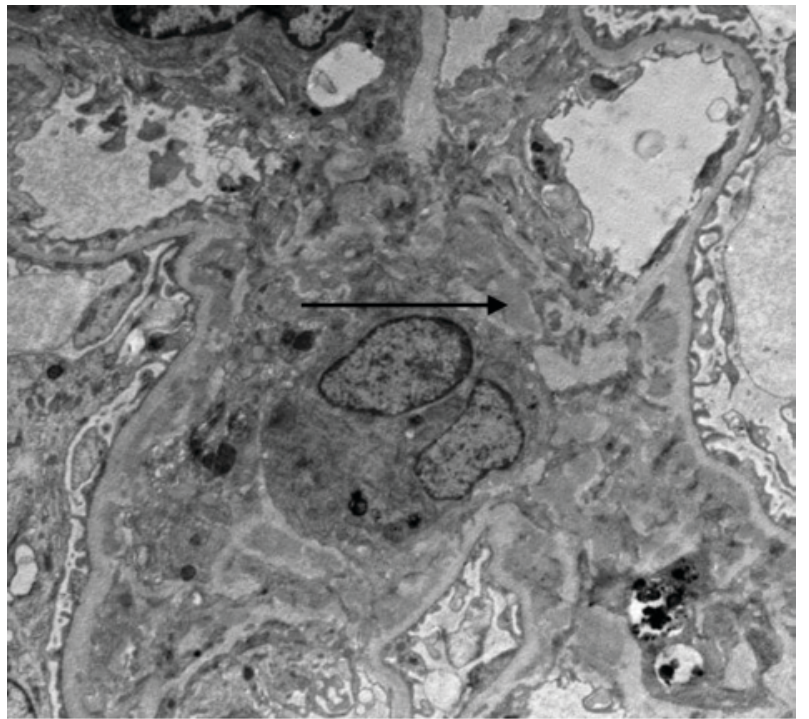

Figure 6. Electron microscopy. The arrow indicates electron-dense deposits in the mesangial area (magnification, x6,000).

complement $\mathrm{C} 4$ and $\mathrm{C} 1 \mathrm{q}$ deposition. $\mathrm{C} 3$ nephropathy is divided into dense deposit disease and $\mathrm{C} 3 \mathrm{GN}$ according to the characteristics on electron microscopy. The electron-dense deposits of $\mathrm{C} 3 \mathrm{GN}$ may be identified in the basement membrane, as well as the mesangial, subendothelial and subepithelial areas $(2,8)$. The histopathological changes in the present case were endocapillary proliferative changes with certain crescentic formatins, C3 granules deposited in the subendothelial and mesangial areas, and electron-dense deposits in mesangial and subendothelial areas. The following diseases were excluded: i) Acute post-streptococcal glomerulonephritis, as the complement remained low at 1 year and there were no subepithelial 
humplike dense deposits observed under electron microscopy; ii) membranoproliferative glomerulonephritis type I, as there was no mesangial cell proliferation, and mesangial cells were not identified in the endothelial cells under light microscopy. In addition, there was only complement C3 deposition and no immunoglobulin deposition observed under immunofluorescence microscope; iii) systemic lupus erythematosus, although the patient had joint swelling and pain, nephritis and low complementemia, the ANA were negative and there was no deposition of various immune complex deposits observed under immunofluorescence microscopy; iv) cryoglobulinemia, the test results were negative for the hepatitis $\mathrm{C}$ antibody, normal for the rheumatoid factor and negative for cryoglobulin in the sera. No thrombus-like substance in the loop cavity was identified; and v) monoclonal Ig disease associated with $\mathrm{C} 3$ nephritis, as the results of chains $\lambda$ in serum, urine $M$ protein and cryoglobulin in the sera were all negative. The bone marrow biopsy did not reveal abnormal plasma cell proliferation. Therefore, the diagnosis of $\mathrm{C} 3 \mathrm{GN}$ was confirmed.

RA-induced GN is rare, and to date, RA with C3GN has not been reported in the relevant literature. In 2016, Alexander et al (5) reported on a cohort of 85 patients diagnosed with C3GN from 2007 to 2014, and of these, 10 patients (3 men and 7 women; 12\%) also had autoimmune diseases. These 10 patients had antinuclear antibody titer abnormalities, and in 6 patients, anti-double-stranded DNA antibodies were detected. The incidence of autoimmune diseases in this previous study was higher than that in the general population. Therefore, $\mathrm{C} 3 \mathrm{GN}$ may be secondary to autoantibodies that directly activate the alternative pathway of $\mathrm{C} 3$ or indirectly lead to its dysregulation. Autoimmune diseases occur early in the course of C3GN. In addition, Alexander et al (5) reported that the treatment was based on the use of prednisone alone or in combination with cyclophosphamide. The serum creatinine and urine protein level decreased significantly, suggesting the presence of C3GN with autoimmune diseases and a good prognosis. The patient of the present study was treated with hormone therapy alone and given maintenance treatment following their diagnosis; the patient's nephritis reached complete clinical remission at 4 months and the condition remained stable after a follow-up of 18 months with low-dose hormone maintenance therapy.

The patient of the present study suffered from kidney damage after years of RA, and at the same time, autoimmune thyroiditis occurred, so the presentation of C3GN may be secondary to autoimmune diseases. However, no direct evidence is available to prove that $\mathrm{C} 3 \mathrm{RN}$ is caused by $\mathrm{RA}$ and/or autoimmune thyroiditis. The patient responded well to hormone therapy and the condition remained stable for a long time, which suggests that the $\mathrm{C} 3 \mathrm{GN}$ may be associated with autoimmune diseases. It should also be noted that the patient's renal histopathological examination indicated the formation of crescents and segmental fibroid necrosis in certain capillary loops, while perinuclear anti-neutrophil cytoplasmic antibody (ANCA) was present; therefore, the combined diagnosis of ANCA-associated vasculitis (AAV) should be considered. A review of the literature indicated that conditions including SLE (9), scleroderma (10) and RA (11), may all occur in combination with AAV, and that the syndromes tend to overlap $(9,11)$. However, the patient had normal renal function and massive proteinuria, immunofluorescence and electron microscopic analysis revealed the deposition of immune complexes, and myeloperoxidase and protease 3 were negative. These results do not support the diagnosis of AAV, and it is therefore unlikely that this patient had AAV.

\section{Acknowledgements}

The authors would like to thank Dr Fan Yang (The Second Hospital of Jilin University, Changchun, China) for capturing the images.

\section{Funding}

No funding was received.

\section{Availability of data and materials}

The analyzed data sets generated during the study are available from the corresponding author on reasonable request.

\section{Authors' contributions}

DZ conceived the present study. XW analyzed the data and prepared the manuscript. XW and LH performed the data analyses and wrote the manuscript. HL assisted with the data analysis.

\section{Ethical approval and consent to participate}

Not applicable.

\section{Patient consent for publication}

The patient provided written informed consent for the publication of their data and associated images.

\section{Competing interests}

The authors declare that they have no competing interests.

\section{References}

1. Fakhouri F, Frémeaux-Bacchi V, Noël LH, Cook HT and Pickering MC: C3 glomerulopathy: A new classification. Nat Rev Nephrol 6: 494-499, 2010

2. Pickering MC, D'Agati VD, Nester CM, Smith RJ, Haas M, Appel GB, Alpers CE, Bajema IM, Bedrosian C, Braun M, et al: C3 glomerulopathy: Consensus report. Kidney Int 84: 1079-1089, 2013.

3. Bridoux F, Desport E, Frémeaux-Bacchi V, Chong CF, Gombert JM, Lacombe C, Quellard $\mathrm{N}$ and Touchard G: Glomerulonephritis with isolated C3 deposits and monoclonal gammopathy: A fortuitous association? Clin J Am Soc Nephrol 6: 2165-2174, 2011 .

4. Zhang LH, Chen Z, Xu F, Zhang T, Zhang HT, Ge YC, Zhou Y, Zeng CH, Hu WX, Liu ZH and Tang Z: C3 glomerulonephritis associated with monoclonal gammopathy. Chin J Nephrol Dial Transplant 24: 507-511, 2015

5. Alexander MP, Fervenza FC, De Vriese AS, Smith RJH, Nasr SH, Cornell LD, Herrera Hernandez LP, Zhang Y and Sethi S: C3 glomerulonephritis and autoimmune disease: More than a fortuitous association? J Nephrol 29: 203-209, 2016.

6. Zhang T, Zhang HT, Xu F, Huang Q, Wang JQ, Zeng CH, Chen HM and Liu ZH: Clinicopathologic features of patients with C3 glomerulopathy. Chin J Nephrol Dial Transplant 23: 426-431, 2014 (In Chinese). 
7. Hou J, Markowitz GS, Bomback AS, Appel GB, Herlitz LC, BarryStokes $M$ and D'Agati VD: Toward a working definition of C3 glomerulopathy by immunofluorescence. Kidney Int 85 : 450-456, 2014.

8. Zhang HT, Chen HP, Zeng CH, Deng KP, Li SJ, Zheng CX and Liu ZH: C3 glomerulonephritis: A clinicopathologic analysis of 17 cases. Chin J Nephrol Dial Transplant 20: 307-311, 2011 (In Chinese).

9. Hervier B, Hamidou M, Haroche J, Durant C, Mathian A and Amoura Z: Systemic lupus erythematosus associated with ANCA-associated vasculitis: An overlapping syndrome? Rheumatology International 32: 3285-3290, 2012.
10. Derrett-Smith EC, Nihtyanova SI, Harvey J, Salama AD and Denton CP: Revisiting ANCA-associated vasculitis in systemic sclerosis: Clinical, serological and immunogenetic factors. Rheumatology (Oxford) 52: 1824-1831, 2013.

11. Draibe J and Salama AD: Association of ANCA associated vasculitis and rheumatoid arthritis: A lesser recognized overlap syndrome. Springerplus 4: 50, 2015. 